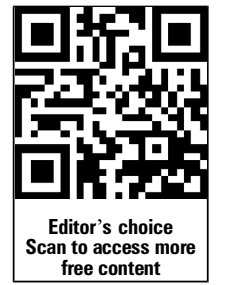

1National Epidemiology C
Carlos III Health Institute,

Madrid, Spain

${ }^{2}$ CIBER of Epidemiology and

Public Health (CIBERESP),

Madrid, Spain

${ }^{3}$ National School of Health.

Carlos III Health Institute,

Madrid, Spain

${ }^{4}$ Network of addictive disorders

(RTA), Madrid, Spain

\section{Correspondence to}

Dr Luis de la Fuente,

National Epidemiology Centre,

Carlos III Health Institute

C/Monforte de Lemos 5,

Madrid 28029, Spain;

Ifuente@isciii.es

Received 4 June 2013

Revised 16 October 2013

Accepted 20 October 2013

Published Online First

14 November 2013

To cite: Fernández-

Balbuena $S$, de la Fuente $L$,

Hoyos J, et al. Sex Transm

Infect 2014;90:112-118.

\title{
Highly visible street-based HIV rapid testing: is it an attractive option for a previously untested population? A cross-sectional study
}

\author{
Sonia Fernández-Balbuena, ${ }_{1,2}^{1,2}$ Luis de la Fuente, ${ }^{1,2}$ Juan Hoyos, ${ }^{1,2}$ \\ M Elena Rosales-Statkus, ${ }^{1,2}$ Gregorio Barrio, ${ }^{3,4}$ María-José Belza, ${ }^{3,2}$ \\ Madrid Rapid HIV testing Group
}

\section{ABSTRACT \\ Background Given the shortage of community-based HIV testing initiatives in resource-rich countries not targeting most-at-risk populations, we aimed to evaluate} whether a highly visible mobile programme promoting and offering rapid HIV testing in the street can attract persons at risk for infection who have never been tested. Methods Between 2008 and 2011, the programme served 7552 persons in various Spanish cities who answered a brief questionnaire while awaiting their results. The factors associated with being tested for the first time were analysed using two logistic regression models, one for men who have sex with men (MSM) and the other for only heterosexual men (MSW) and women.

Results 3517 participants (47\%) were first-time testers ( $24 \%$ of MSM, $56 \%$ of MSW and $60 \%$ of women). Among them, 22 undiagnosed HIV infections were detected with a global prevalence of $0.6 \%$ and $3.1 \%$ in MSM. Undergoing a first HIV test was independently associated with age $<30$, being from Spain or another developed country, lack of university education, having fewer partners, having had unprotected sex with casual partners and not having been diagnosed with a sexually transmitted infection. In heterosexuals, also with never injected drugs, and in MSM, with not being involved in the gay community. Among those tested for the first time, $22 \%$ had never thought of being tested and $62 \%$ decided to be tested when they passed by and noticed the programme, regardless of their previous intentions. Conclusions This community programme attracted a substantial number of persons previously untested and particularly hard to reach, such as those with low education and MSM who were least involved in the gay community. Programme visibility was a decisive factor for almost two of every three persons who had never been tested.

\section{INTRODUCTION}

Lacking a cure or an effective vaccine for HIV, screening strategies are critical to reduce the number of undiagnosed persons and the proportion of late diagnoses in order to modify the course of the epidemic. ${ }^{1}{ }^{2}$ Consequently, facilitating access to HIV testing to increase its coverage is a priority in developed countries. In 2006, the US Centres for Disease Control and Prevention proposed a radical change in its testing strategy by recommending standardised, non-targeted testing for persons aged
13-64 years in all healthcare settings. ${ }^{3}$ In 2010, its European counterpart, the European Centre for Disease Control, recommended the promotion of strategies aimed at key populations at risk to enable early diagnosis of infection. ${ }^{4}$

In this direction, one of the most widespread initiatives in recent years has been the implementation of rapid testing programmes in non-clinical settings, conducted mostly, but not exclusively, by community-based organisations (CBOs). These types of services are a good model for improving access to the test in the most vulnerable and difficult-to-reach populations because they eliminate many of the usual barriers present in the traditional health services. ${ }^{4}$

Many different kinds of programmes currently promote the test. Some serve especially vulnerable populations (drug injectors, immigrants, etc), while others focus their activities on the general population, serving all who perceive themselves to be at risk and request their services. ${ }^{5}$

Most of these programmes are located indoors, on the premises of CBOs, and some have developed different types of outreach services ${ }^{4}$ offering locations that are more attractive and accessible than conventional health settings.

In Europe, most evaluations of programmes offering rapid testing in non-clinical settings have been conducted in the UK, in programmes that primarily target men who have sex with men (MSM) and usually located in indoor CBO settings. ${ }^{6}$ However, there is little in the scientific literature about the evaluation of outreach programmes that do not target the most-at-risk groups, therefore no studies have explored whether their visibility can spark the interest of a population that, despite engaging in risk behaviours, has never considered being tested.

Our aim was to evaluate whether a highly visible mobile programme promoting and offering rapid HIV testing, anonymously and free of charge, can attract persons who are at risk for infection but have never been tested.

\section{METHODS}

Between May 2008 and March 2011, a mobile unit (van) offered free, rapid HIV testing, mainly in the city of Madrid, but also in two dormitory towns outside Madrid, as well as three cities on Spain's south-eastern coast and in the Canary Islands. The 
van was always located near railway stations or on centrally located streets with high pedestrian traffic, attempting to be as visible as possible and with some posters announcing the test.

In a tent outside the van, an educator provided interested passersby with brief counselling about how the rapid test is performed and its limitations. Those who decided to proceed with testing signed an informed consent form and entered the van, where medical or nursing staff completed the counselling and performed the test by taking a finger stick capillary blood sample. While awaiting their results, participants completed an anonymous questionnaire on paper which was linked by a code to the test results. Since no place was provided to answer the questionnaire and most people filled it out while standing in the street, it was decided to limit its length. Various versions of the questionnaire were made during the course of the programme; to keep its length constant, some questions were not always included throughout the entire programme. Information was collected on sociodemographics, sexual behaviour and history of HIV test performance. For those with limited proficiency in Spanish, a form was designed to collect the basic sociodemographic and behaviour data in English and French.

Those with reactive results were referred to a collaborating HIV/sexually transmitted infection (STI) diagnostic centre or advised to see their primary care physician. Starting in 2009 and onwards, blood was extracted at the van and sent to a collaborating STI diagnostic centre for confirmation.

\section{Data analysis}

A descriptive analysis was made stratifying the population into three groups based on their gender and sexual behaviour; the latter was understood to refer to the type of sexual relations during the person's lifetime: MSM, men who were exclusively heterosexual (MSW) and women. Persons who did not provide information about their sexual behaviour or history of HIV testing were excluded from the analysis (a total of 524). When the version of the questionnaire did not include a certain question, data were labelled as 'not in questionnaire' and the percentages were calculated on respondents.

A bivariate analysis was performed to identify factors associated with not having had a previous HIV test. To estimate the magnitude and precision of the associations, we calculated ORs and $95 \%$ CIs (95\% CI). Following the methodology proposed by Hosmer and Lemeshow ${ }^{7}$, we then built three different logistic regression models, one for each category of gender/sexual behaviour. Due to the similarities between the models for women and MSW, however, it was decided to build only two models: one for MSM and the other combining MSW and women. The models initially included the variables that were associated with never having had an HIV test in the bivariate analysis at a significance level of $p<0.2$. To assess the contribution of each variable to the model, we used the likelihood-ratio test. Non-significant variables were excluded from the final models.

To classify participants according to their risk behaviours for HIV infection (taking into consideration the Spanish epidemic), ${ }^{8}$ we created an indicator that classified them as high risk if they had ever injected drugs, engaged in sex work or had not always used condoms with casual partners in the previous 12 months.

The study was approved by the institutional review board of the Carlos III Health Institute.

\section{RESULTS}

\section{Sociodemographic and behavioural characteristics}

Of the 7552 persons who participated in the study, 34\% were MSM, 31\% were MSW and 35\% were women. Over half were older than 30 years, and the women were on average younger than the men $(63.7 \%$ of women were under 30 vs $48.8 \%$ of MSW and 49.2\% of MSM). Most were Spanish (68.7\%), and Latin Americans made up the largest group (21.1\%) of those who were foreign-born. Although almost half of the participants had university education, this percentage was much lower in the case of MSW $(39.9 \%$ vs $54.6 \%$ of MSM and $50.6 \%$ of women). Among MSM, although 78.5\% identified themselves as homosexual, $25 \%$ stated they were not involved in the gay culture (table 1).

Only $3 \%$ had ever injected drugs. In the previous 12 months, $22.9 \%$ of MSM had had unprotected sexual relations with casual partners as compared with $34.8 \%$ of MSW and 39\% of women. In the same period, over half of MSM reported having had relations with five or more partners versus $26.2 \%$ of MSW and $17.1 \%$ of women. The internet was the main source of meeting sexual partners for $24.1 \%$ of MSM. Around $59.2 \%$ had never been diagnosed with an STI, although there were large differences among MSM (45.9\%), MSW (65.9\%) and women $(66.3 \%)$. (table 1$)$

\section{HIV testing}

Some $46.6 \%$ of participants had never undergone an HIV test before. This proportion was less frequent in MSM (24.2\%) than in MSW (56.1\%) or women (59.8\%), with virtually no difference between the latter two groups (table 1).

Of those 3517 first-time testers, 22 were positive (prevalence of undiagnosed HIV infection: 0.6\%): 19 MSM (3.1\%), 1 MSW $(0.1 \%)$ and 2 women $(0.1 \%)$.

In the multivariate analysis, the factors associated with being first-time testers were, in both groups: age under 30 years, having been born in Spain or another developed country, lack of university education, having had unprotected sexual relationships with casual partners in the last 12 months and never having been diagnosed with an STI. Furthermore, the risk of not having been tested previously for HIV increased with decreasing number of sexual partners in the last year. In the case of MSW and women, it was also associated with never having used intravenous drugs. In MSM, those who were not related to gay scene were more likely never to have been tested than those who were member of a gay CBO (table 2).

Among first-time testers, $21.6 \%$ reported not being at all concerned in this respect, with clear differences between MSM (10.9\%) and MSW and women (24.9\% and 23.3\%, respectively). About $46.6 \%$ said they had intended to take the test in the next 12 months $(63.3 \%$ of MSM, 48.2\% of MSW and $39.3 \%$ of women). However, $61.8 \%$ said that if they had not passed by the mobile unit they would not have been tested (table 3).

The question regarding the reasons for testing in this particular programme was available in the questionnaire for 15 out of the 22 first-time testers who were positive. Of them, four MSM and one woman said that if they had not passed by the mobile unit, they would not have been tested (33.3\%).

In the analysis of risk behaviours, $47.9 \%$ of persons who had never been tested for HIV were classified as persons with a high risk of infection (48.3\% of MSM, 48.2\% of MSW and 58\% of women). Of these, $7 \%$ of MSM stated they had never been concerned or thought about taking the test, and 28\% said they had been thinking about it for 2 years or more. In contrast, high-risk MSW and women with no previous test were three times more likely to express a complete lack of concern $(22.5 \%$ and $17.3 \%$, respectively) than were MSM (figure 1). 
Table 1 Sociodemographic and behavioural characteristics of participants in a street-based rapid HIV testing programme in Spain (2008-2011)

\begin{tabular}{|c|c|c|c|c|c|c|c|c|}
\hline & \multicolumn{2}{|c|}{$\begin{array}{l}\text { MSM } \\
(\mathrm{N}=2559)\end{array}$} & \multicolumn{2}{|c|}{$\begin{array}{l}\text { MSW } \\
(\mathrm{N}=2326)\end{array}$} & \multicolumn{2}{|c|}{$\begin{array}{l}\text { Women } \\
(\mathrm{N}=2667)\end{array}$} & \multicolumn{2}{|c|}{$\begin{array}{l}\text { Total } \\
(\mathrm{N}=7552)\end{array}$} \\
\hline & $\mathbf{N}$ & $\%$ & $\mathrm{~N}$ & $\%$ & $\mathbf{N}$ & $\%$ & $\mathbf{N}$ & $\%$ \\
\hline \multicolumn{9}{|l|}{ Sociodemographic } \\
\hline \multicolumn{9}{|l|}{ Age } \\
\hline Under 25 & 635 & 25.3 & 505 & 22.6 & 919 & 36.0 & 2059 & 28.2 \\
\hline $25-29$ years & 598 & 23.9 & 584 & 26.2 & 709 & 27.7 & 1891 & 25.9 \\
\hline 30 years or older & 1273 & 50.8 & 1144 & 51.2 & 928 & 36.3 & 3345 & 45.9 \\
\hline \multicolumn{9}{|l|}{ Country of origin } \\
\hline Spain & 1736 & 69.9 & 1528 & 67.5 & 1783 & 68.7 & 5047 & 68.7 \\
\hline Western Europe, North America and other developed countries & 162 & 6.5 & 123 & 5.4 & 154 & 5.9 & 439 & 6.0 \\
\hline Latin America & 536 & 21.6 & 455 & 20.1 & 562 & 21.6 & 1553 & 21.1 \\
\hline Other developing countries & 50 & 2.0 & 158 & 7.0 & 98 & 3.8 & 306 & 4.2 \\
\hline With university education & 1380 & 54.6 & 910 & 39.9 & 1332 & 50.6 & 3622 & 48.6 \\
\hline Employment as main source of income & 2095 & 84.6 & 1856 & 86.2 & 2011 & 78.6 & 5962 & 83.0 \\
\hline \multicolumn{9}{|l|}{ Self-reported sexual orientation } \\
\hline Homosexual & 1117 & 78.5 & & & & & & \\
\hline Bisexual & 165 & 11.6 & & & & & & \\
\hline Heterosexual & 141 & 9.9 & & & & & & \\
\hline Not in questionnaire* & 1057 & & & & & & & \\
\hline \multicolumn{9}{|l|}{ Relationship with gay culture } \\
\hline Member of a gay CBO & 178 & 11.1 & & & & & & \\
\hline Frequents gay scene, not member of a gay $C B O$ & 1023 & 63.9 & & & & & & \\
\hline Not related to gay scene & 400 & 25.0 & & & & & & \\
\hline Not in questionnaire* & 684 & & & & & & & \\
\hline \multicolumn{9}{|l|}{ Behavioural } \\
\hline Ever injected drugs & 67 & 2.7 & 102 & 4.6 & 49 & 1.9 & 218 & 3.0 \\
\hline Unprotected sex with casual partnerst’ & 585 & 22.9 & 809 & 34.8 & 1039 & 39.0 & & \\
\hline \multicolumn{9}{|l|}{ Number of heterosexual partners $\dagger$} \\
\hline $0-1$ & & & 574 & 27.6 & 962 & 39.3 & & \\
\hline $2-4$ & & & 962 & 46.2 & 1068 & 43.6 & & \\
\hline 5 or more & & & 546 & 26.2 & 419 & 17.1 & & \\
\hline \multicolumn{9}{|l|}{ Number of homosexual partners $\dagger$} \\
\hline $0-1$ & 483 & 19.9 & & & & & & \\
\hline $2-4$ & 691 & 28.4 & & & & & & \\
\hline $5-9$ & 487 & 20.0 & & & & & & \\
\hline 10 or more & 768 & 31.6 & & & & & & \\
\hline \multicolumn{9}{|l|}{ Self-reported STI } \\
\hline Never diagnosed with a STI & 923 & 45.9 & 1188 & 65.9 & 1383 & 66.3 & 3494 & 59.2 \\
\hline Diagnosed within the last 12 months & 240 & 12.0 & 68 & 3.8 & 166 & 8.0 & 474 & 8.0 \\
\hline Not in questionnaire* & 549 & & 524 & & 581 & & 1654 & \\
\hline Internet as main source of meeting partners $†$ & 362 & 24.1 & & & & & & \\
\hline Not in questionnaire* & 1057 & & & & & & & \\
\hline \multicolumn{9}{|l|}{ History of HIV testing } \\
\hline Never tested & 619 & 24.2 & 1304 & 56.1 & 1594 & 59.8 & 3517 & 46.6 \\
\hline Tested less than a year ago & 831 & 32.5 & 297 & 12.8 & 251 & 9.4 & 1379 & 18.3 \\
\hline Tested between 1 and 3 years ago & 604 & 23.6 & 298 & 12.8 & 351 & 13.2 & 1253 & 16.6 \\
\hline Tested between 3 and 5 years ago & 117 & 4.6 & 97 & 4.2 & 139 & 5.2 & 353 & 4.7 \\
\hline Tested over 5 years ago & 141 & 5.5 & 126 & 5.4 & 150 & 5.6 & 417 & 5.5 \\
\hline
\end{tabular}

*For some time, this question was not available in all questionnaires. Percentages calculated on respondents.

tIn the last 12 months.

¥Anal intercourse when referring to MSM; anal/vaginal intercourse when referring to women and MSW.

CBO, community-based organisation; MSM, men who have sex with men; MSW, men who have sex only with women; STI, sexually transmitted infection.

\section{DISCUSSION}

This is one of the first published studies to evaluate the ability of an outreach programme offering rapid HIV testing not aimed at the most-at-risk populations to attract persons with risk practices for infection who have never been tested. The high visibility of the programme-given that it was conducted in the street in very busy areas-was a decisive factor in the decision to be tested for almost two-thirds of first-time testers.

Our results show that almost half of the persons served by the programme were undergoing testing for the first time. Comparison of our results with those of other authors is difficult because few studies have been made of outreach 
Table 2 Crude and adjusted association with not having undergone an HIV test before among participants in a street-based rapid HIV testing programme in Spain (2008-2011)

\begin{tabular}{|c|c|c|c|c|c|c|c|c|c|c|}
\hline & \multicolumn{5}{|c|}{ Men who have sex with men (MSM) } & \multicolumn{5}{|c|}{ Men who are exclusively heterosexual and women } \\
\hline & $\%$ & $\mathrm{OR}_{\mathrm{c}}$ & $(95 \% \mathrm{Cl})$ & $\mathrm{OR}_{\mathrm{a}}$ & $(95 \% \mathrm{Cl})$ & $\%$ & $\mathrm{OR}_{\mathrm{c}}$ & $(95 \% \mathrm{Cl})$ & $\mathrm{OR}_{\mathrm{a}}$ & $(95 \% \mathrm{Cl})$ \\
\hline \multicolumn{11}{|l|}{ Age } \\
\hline$\geq 30$ years & 15.1 & 1.0 & & 1.0 & & 47.9 & 1.0 & & 1.0 & \\
\hline$<30$ years & 33.3 & 2.8 & (2.3 to 3.4$)$ & 2.7 & (2.2 to 3.3 ) & 66.1 & 2.1 & (1.9 to 2.4$)$ & 2.0 & (1.8 to 2.3$)$ \\
\hline \multicolumn{11}{|l|}{ Country of origin } \\
\hline Latin America and other developing countries & 18.6 & 1.0 & & 1.0 & & 46.3 & & & 1.0 & \\
\hline Spain and other developed countries & 26.2 & 1.6 & $(1.2$ to 2.0$)$ & 1.6 & $(1.2$ to 2.0$)$ & 62.5 & 1.0 & (1.7 to 2.2$)$ & 1.9 & (1.6 to 2.1$)$ \\
\hline \multicolumn{11}{|l|}{ With university education } \\
\hline Yes & 20.5 & 1.0 & & 1.0 & & 53.5 & 1.0 & & 1.0 & \\
\hline No & 28.5 & 1.5 & (1.3 to 1.9$)$ & 1.3 & (1.1 to 1.6$)$ & 61.9 & 1.4 & (1.3 to 1.6$)$ & 1.4 & $(1.3$ to 1.6$)$ \\
\hline \multicolumn{11}{|l|}{ Ever injected drugs } \\
\hline Yes & 20.9 & 1.0 & & & & 43.0 & 1.0 & & 1.0 & \\
\hline No & 24.8 & 1.2 & (0.7 to 2.3 ) & & & 59.3 & 1.9 & (1.4 to 2.7$)$ & 2.0 & (1.4 to 2.8$)$ \\
\hline \multicolumn{11}{|l|}{ Self-reported sexual orientation } \\
\hline Homosexual & 19.1 & & & & & & & & & \\
\hline Heterosexual & 36.9 & 1.0 & (1.7 to 3.6$)$ & & & & & & & \\
\hline Bisexual & 39.4 & 2.8 & (2.0 to 3.9$)$ & & & & & & & \\
\hline Not in questionnaire* & 25.4 & 1.4 & (1.2 to 1.8$)$ & & & & & & & \\
\hline \multicolumn{11}{|l|}{ Relationship with gay culture } \\
\hline Member of a gay CBO & 16.9 & 1.0 & & 1.0 & & & & & & \\
\hline Frequents gay scene, not member of a gay $C B O$ & 19.3 & 1.2 & (0.8 to 1.8$)$ & 1.1 & (0.7 to 1.8$)$ & & & & & \\
\hline Not related to gay scene & 32.0 & 2.3 & (1.5 to 3.6$)$ & 1.9 & $(1.2$ to 3.1$)$ & & & & & \\
\hline Not in questionnaire* & 26.0 & 1.7 & (1.1 to 2.7$)$ & 1.4 & (0.9 to 2.2$)$ & & & & & \\
\hline \multicolumn{11}{|c|}{ Unprotected sex with casual partners in the last 12 months $t$} \\
\hline No & 22.1 & 1.0 & & 1.0 & & 58.8 & 1.0 & & 1.0 & \\
\hline Yes & 25.1 & 1.2 & (1.0 to 1.4$)$ & 1.4 & $(1.2$ to 1.8$)$ & 57.6 & 1.0 & (0.8 to 1.1$)$ & 1.2 & $(1.0$ to 1.4$)$ \\
\hline \multicolumn{11}{|c|}{ Number of heterosexual partners in the last 12 months } \\
\hline$\geq 5$ & & & & & & 48.2 & 1.0 & & 1.0 & \\
\hline 2 to 4 & & & & & & 56.7 & 1.4 & (1.2 to 1.6$)$ & 1.5 & $(1.2$ to 1.7$)$ \\
\hline 0 to 1 & & & & & & 66.7 & 2.2 & (1.8 to 2.5$)$ & 2.4 & (2.0 to 2.9$)$ \\
\hline \multicolumn{11}{|l|}{ Number of homosexual partners in the last 12 months } \\
\hline$\geq 10$ & 15.5 & 1.0 & & 1.0 & & & & & & \\
\hline 5 to 9 & 21.8 & 1.5 & (1.1 to 2.0$)$ & 1.3 & (1.0 to 1.8$)$ & & & & & \\
\hline 2 to 4 & 27.6 & 2.1 & (1.6 to 2.7$)$ & 1.7 & (1.3 to 2.0$)$ & & & & & \\
\hline 0 to 1 & 35.8 & 3.0 & (2.3 to 4.0$)$ & 2.5 & (1.8 to 3.4$)$ & & & & & \\
\hline \multicolumn{11}{|c|}{ Internet as main source of meeting partners in the last 12 months } \\
\hline No & 20.9 & 1.0 & & & & & & & & \\
\hline Yes & 19.3 & 0.9 & (0.7 to 1.3$)$ & & & & & & & \\
\hline Not in questionnaire* & 25.6 & 1.3 & (1.0 to 1.7$)$ & & & & & & & \\
\hline \multicolumn{11}{|l|}{ Self-reported STI } \\
\hline Ever been diagnosed & 14.1 & 1.0 & & 1.0 & & 44.4 & 1.0 & & 1.0 & \\
\hline Never & 36.0 & 3.4 & (2.7 to 4.3 ) & 2.7 & (2.1 to 3.4 ) & 62.3 & 2.1 & (1.7 to 2.5 ) & 1.8 & (1.5 to 2.1 ) \\
\hline Not in questionnaire* & 20.8 & 1.6 & (1.2 to 2.1$)$ & 1.5 & (1.1 to 2.1$)$ & 57.6 & 1.7 & (1.4 to 2.1$)$ & 1.6 & (1.3 to 1.9$)$ \\
\hline
\end{tabular}

$\%$, percentage that have never been tested.

*For some time, this question was not available in all questionnaires.

†Anal intercourse when referring to MSM; anal/vaginal intercourse when referring to women and men who are exclusively heterosexual.

$\mathrm{CBO}$, community-based organisation; $\mathrm{OR}_{\mathrm{a}}$, adjusted $\mathrm{OR} ; \mathrm{OR}_{\mathrm{c}}$ crude $\mathrm{OR}$; $\mathrm{STI}$, sexually transmitted infection.

programmes that do not target a specific population group. ${ }^{6}$ However, our results are consistent with the scant literature available on non-targeted programmes. ${ }^{9-12}$ The notable difference found among the three exposure categories (MSM, MSW and women) in the proportion of first-time testers is consistent with the findings of other studies that identify MSM as the population group that is tested in the largest proportion and with greatest frequency. ${ }^{13-16}$ This is even more evident in the analysis of data from non-clinical settings. ${ }^{6}$
Among the factors associated with a higher probability of not having been tested, age and low educational level are involved in several ways: younger people have had fewer opportunities to be tested during their lifetimes and also have fewer sexual partners. ${ }^{15} 1718$ Age takes on additional importance in the case of women, because prenatal screening is the main factor for them being tested, ${ }^{14}{ }^{19}$ and younger women are less likely to have been screened in this way. Low educational level is usually associated with less perceived risk of 
Table 3 Level of concern, intention to undergo HIV testing and reason for choosing a street-based rapid HIV testing programme among persons being tested for the first time

\begin{tabular}{|c|c|c|c|c|c|c|c|c|}
\hline & \multicolumn{2}{|c|}{$\begin{array}{l}\text { MSM } \\
(\mathrm{N}=619)\end{array}$} & \multicolumn{2}{|c|}{$\begin{array}{l}\text { MSW } \\
(\mathrm{N}=1304)\end{array}$} & \multicolumn{2}{|c|}{$\begin{array}{l}\text { Women } \\
(\mathrm{N}=1594)\end{array}$} & \multicolumn{2}{|c|}{$\begin{array}{l}\text { Total } \\
(\mathrm{N}=3517)\end{array}$} \\
\hline & $\mathbf{N}$ & $\%$ & $\mathbf{N}$ & $\%$ & $\mathbf{N}$ & $\%$ & $\mathbf{N}$ & $\%$ \\
\hline \multicolumn{9}{|l|}{ Thoughts about undergoing an HIV test } \\
\hline Was never concerned or thought about it & 58 & 10.9 & 257 & 24.9 & 313 & 23.3 & 628 & 21.6 \\
\hline Has been thinking about it for less than 6 months & 160 & 30.2 & 237 & 23.0 & 330 & 24.5 & 727 & 25.0 \\
\hline Has been thinking about it between 6 months and $<1$ year & 82 & 15.5 & 125 & 12.1 & 163 & 12.1 & 370 & 12.7 \\
\hline Has been thinking about it between 1 and $<2$ years & 89 & 16.8 & 170 & 16.5 & 227 & 16.9 & 486 & 16.7 \\
\hline Has been thinking about for 2 years or more & 141 & 26.6 & 243 & 23.5 & 313 & 23.3 & 697 & 24.0 \\
\hline \multicolumn{9}{|c|}{ If you hadn't taken the test today, would you have in the next 12 months? } \\
\hline No or probably no & 60 & 18.8 & 220 & 32.4 & 351 & 39.6 & 631 & 33.5 \\
\hline I'm not sure & 57 & 17.9 & 132 & 19.4 & 187 & 21.1 & 376 & 19.9 \\
\hline Yes or probably yes & 202 & 63.3 & 328 & 48.2 & 349 & 39.3 & 879 & 46.6 \\
\hline Not in questionnaire* & 283 & & 506 & & 635 & & 1424 & \\
\hline \multicolumn{9}{|l|}{ Reasons for testing in this particular programme } \\
\hline I knew how it worked and I came expressly to be tested & 174 & 36.1 & 328 & 36.0 & 402 & 34.0 & 904 & 35.1 \\
\hline If I hadn't passed by here, I wouldn't have done it & 295 & 61.2 & 548 & 60.2 & 750 & 63.4 & 1593 & 61.8 \\
\hline Other & 13 & 2.7 & 35 & 3.8 & 31 & 2.6 & 79 & 3.1 \\
\hline Not in questionnaire* & 114 & & 286 & & 357 & & 757 & \\
\hline
\end{tabular}

infection and, consequently, with less likelihood of undergoing HIV testing. ${ }^{15} 1718$

Persons with no or only one sexual partner in the last 12 months and who had never been diagnosed with an STI were, logically, more likely never to have been tested. This makes sense considering that persons with fewer risk behaviours are less likely to undergo a test. Also, in women and MSW, not having used injected drugs was related with never having been tested. In both models, we found that those who had never been tested were more likely to have engaged in unprotected sex with casual partners. This is consistent with the findings of other studies, ${ }^{21}$ although some authors have not found any association, ${ }^{15}$ while others have reported an inverse association in $\mathrm{MSM}^{22}$ that is, that those not previously tested were less likely to have engaged in unprotected sex.

Our findings show that almost half of the first testers had high-risk behaviours for HIV transmission. Most studies agree that the lack of perceived risk is the main barrier to never being tested. ${ }^{4}$ In fact, a recent study in Spain reported that slightly more than half of persons with no previous test reported

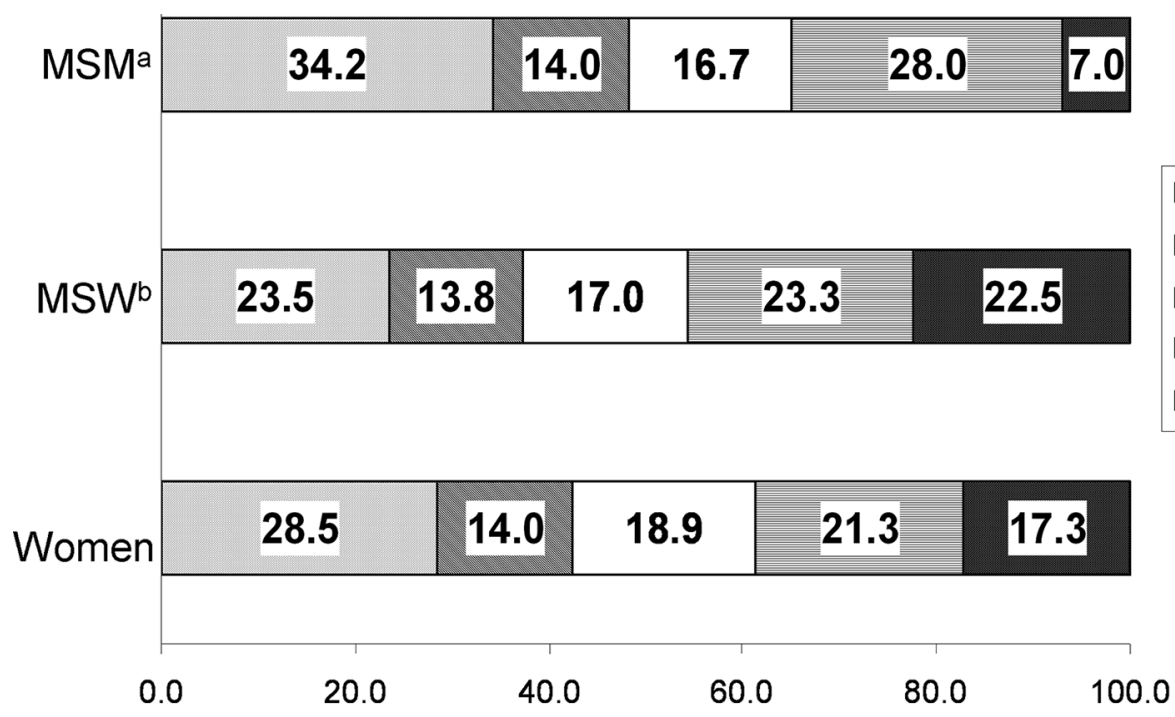

a MSM: Men who have sex with men $\quad$ b MSW: Men who have sex only with women

*High-risk people for HIV: ever injected drugs, engaged in prostitution, or had not always used condoms with casual partners in the previous 12 months

Figure 1 Thoughts about undergoing an HIV test for the first time among high-risk people* who get tested in a street-based rapid HIV testing programme in Spain (2008-2011). 
reasons related with low-risk perception. ${ }^{23}$ It may be that, in casual sex encounters, untested people rely on strategies of questionable safety such as selection of partners based on appearance, level of trust, romantic feelings, discussion of each partner's sexual history or their belief that those who have previously tested negative will disclose this circumstance so they can assess the risk of HIV transmission. ${ }^{22}{ }^{24}$ The results of this study indicate that persons at highest risk for HIV, based on the report of sexual risk behaviour, are not being reached by HIV testing services, since those who had never been tested for HIV were more likely to report unprotected sex with casual partners.

It is important not to overlook the fact that, in the case of MSM, the level of involvement in the gay community predicts the likelihood of not being tested: the lower the involvement in the gay culture, the greater the probability of not being tested, as has been reported previously. ${ }^{15} 22$ This association is so strong that, in our case, the probability of never having been tested in those not involved in the gay community is double that of those who are members of a CBO dedicated to defending the rights of homosexuals. It is quite obvious that this especially at-risk population is not going to be reached by interventions that focus on the test promotion within the gay community. Accordingly, public health policies need to consider this 'hidden' population group that is difficult to access and recruit, but which constitutes a non-negligible percentage of all MSM ( $25 \%$ in our study).

It should also be emphasised that the high visibility of the programme was the most important factor for undergoing testing in nearly two-thirds of those with no previous test and for one-third of the first testers who tested positive. Whereas lack of time or interest and not knowing where to be tested are frequently cited as impediments to testing, outreach programmes can overcome these barriers by providing the opportunity to passersby. This adds value to the programme, especially considering that one out of four MSW (the group with the largest proportion of delayed diagnoses) classified as being at high risk for HIV infection and who received their first test our programme were almost entirely unconcerned about the matter. Furthermore, in the case of MSM (the group most affected by the epidemic throughout Western Europe) who had never been tested but had engaged in risk behaviours, one of every three reported having been thinking about doing it for over 2 years or not having thought about it at all. With such a low level of concern and so little interest in being tested, we believe that, without the participation of easily accessible and highly visible outreach programmes like ours, it will be difficult to meet international guideline recommendations that certain groups such as MSM and persons with multiple sexual partners should be tested at least once a year. ${ }^{25-27}$ It is important to note that this gap will be even greater if the frequency of recommended testing is increased, as appears likely. ${ }^{28}{ }^{29}$ Furthermore, taking the first HIV test, as long as it is a pleasant experience (usually the case in programmes outside clinical settings ${ }^{6}$ ) should favour subsequent testing, since it helps to create the habit, eliminate fears and distrust and awaken the awareness of risk. If we take into consideration also that one-third of the first testers diagnosed would not have tested if they had not seen the mobile unit, the extreme visibility of the programme takes on even to greater prominence.

The findings in this report are subject to several limitations that should be considered with regard to the generalisation of the results. Since this population was recruited in a rapid HIV testing service, participation may have been biased by the same factors that keep some people from seeking the test. Thus, we need to know the opinions and characteristics of those persons who, despite having risk behaviours for HIV infection, have never been tested and whose recruitment in this type of service would, obviously, not be feasible.

In conclusion, this study has made it possible to determine the ability of a street-based outreach programme of rapid HIV testing not aimed at any particular risk group to attract at-risk and hard-to-reach individuals who have never been tested. This outdoor outreach service is characterised by low threshold HIV testing in that it offers multiple and more attractive, accessible and convenient testing locations than conventional healthcare settings. Thus, in addition to other existing HIV testing services, it may increase access to HIV testing by high-risk persons unaware of their HIV serostatus.

\section{Key messages}

- This street-based outreach rapid HIV testing programme not targeting a particular risk group was able to attract and diagnose previously untested and at-risk individuals.

- The high visibility of the programme has triggered testing in a substantial proportion of the attendees and also of those diagnosed.

- This may increase access to HIV testing to some hard-to-reach populations such as MSM not related to the gay scene.

Handling editor Jackie A Cassell

Collaborators Madrid Rapid HIV testing Group: Jorge Gutierrez, Mónica Ruiz, Rebeca Sánchez and Enrique Regidor.

Contributors MJB, LdelaF and SFB designed the study and wrote the protocol. MJB and LdelaF took overall responsibility for the project. MER-S, GB and the Madrid Rapid HIV testing Group made contributions to the study design, data collection tools, pilot study and participated in the acquisition of data. SFB and JH did the analysis and interpretation of data. SFB, LdelaF and JH wrote the manuscript. MJB critically revised the manuscript and contributed important intellectual content. All authors reviewed, edited and approved the manuscript.

Funding This study was supported by FIPSE 240961/10 and FIPSE 36634/07. The founding source was not involved in the study design; data collection, analysis, interpretation; in the writing of the report or in the decision to submit the paper for publication. All the authors declare not having any competing interests.

\section{Competing interests None.}

Ethics approval The institutional review board of the Instituto de Salud Carlos III. Provenance and peer review Not commissioned; externally peer reviewed.

Data sharing statement Some data were presented previously at XV Spanish AIDS Conference (abstract reference: P3.11) Madrid 2012.

\section{REFERENCES}

1 European Centre for Disease Prevention and Control. Novel approaches to testing for sexually transmitted infections, including HIV and hepatitis B and C in Europe. Stockholm: ECDC, 2012.

2 WHO Regional Office for Europe. Scaling up HIV testing and counselling in the WHO European Region as an essential component of efforts to achieve universal access to HIV prevention, treatment, care and support. Copenhagen: World Health Organization Europe, 2010.

3 Branson BM, Handsfield HH, Lampe MA, et al. Revised recommendations for HIV testing of adults, adolescents, and pregnant women in health-care settings. MMWR Recomm Rep 2006;55:1-17.

4 European Centre for Disease Prevention and Control. HIV testing: increasing uptake and effectiveness in the European Union. Stockholm, 2010.

5 Breveglieri M, Furegato M, Agusti C, et al. The HIV-COBATEST project: cross-national survey on the implementation of CBVCT programmes-quantitative report. HIV-COBATEST, 2012. 
6 Thornton AC, Delpech V, Kall MM, et al. HIV testing in community settings in resource-rich countries: a systematic review of the evidence. HIV Med 2012;13:416-26.

7 Hosmer DW, Lemeshow S. Applied Logistic Regression, second edition. 2 ed. New York: John Wiley \& Sons Inc., 2000.

8 Centro Nacional de Epidemiología. Vigilancia Epidemiológica del VIH/SIDA en España. Sistema de información sobre Nuevos Diagnósticos de VIH y Registro Nacional de SIDA. Actualización 30 junio de 2012. 2012.

9 de la Fuente L, Delgado J, Hoyos J, et al. Increasing early diagnosis of HIV through rapid testing in a street outreach program in Spain. AIDS Patient Care STDS 2009:23:625-9.

10 DiFranceisco W, Holtgrave DR, Hoxie N, et al. HIV seropositivity rates in outreach-based counseling and testing services: program evaluation. J Acquir Immune Defic Syndr Hum Retrovirol 1998;19:282-8.

11 Gorostiza I, Lopez dL I, Braceras IL. HIVIAIDS screening program in community pharmacies in the Basque Country (Spain). Gac Sanit 2013;27:164-6.

12 Guenter D, Greer J, Barbara A, et al. Rapid point-of-care HIV testing in community-based anonymous testing program: a valuable alternative to conventional testing. AIDS Patient Care STDS 2008;22:195-204.

13 Centers for Disease Control and Prevention. Vital Signs: HIV testing and diagnosis among adults-United States, 2001-2009. MMWR Morb Mortal Wkly Rep 2010;59:1550-5.

14 de la Fuente L, Suarez M, Belza MJ, et al. Human immunodeficiency virus testing uptake and risk behaviours in Spain. J Epidemiol Community Health 2009;63:552-8.

15 Margolis $A D$, Joseph $H$, Belcher $L$, et al. 'Never testing for HIV' among men who have sex with men recruited from a sexual networking website, United States. AIDS Behav 2012;16:23-9.

16 Jenness SM, Murrill CS, Liu KL, et al. Missed opportunities for HIV testing among high-risk heterosexuals. Sex Transm Dis 2009;36:704-10.

17 Burns F, Fenton KA, Morison L, et al. Factors associated with HIV testing among black Africans in Britain. Sex Transm Infect 2005;81:494-500.

18 Dias S, Gama A, Severo M, et al. Factors associated with HIV testing among immigrants in Portugal. Int J Public Health 2011;56:559-66.
19 Conaty SJ, Cassell JA, Harrisson U, et al. Women who decline antenatal screening for HIV infection in the era of universal testing: results of an audit of uptake in three London hospitals. J Public Health (Oxf) 2005:27:114-17.

20 Southgate J, Mital D, Stock A. Are women from high-risk ethnic minority groups more likely to decline antenatal HIV screening? Int J STD AIDS 2008:19:206-7.

21 Li X, Lu H, Raymond HF, et al. Untested and undiagnosed: barriers to HIV testing among men who have sex with men, Beijing, China. Sex Transm Infect 2012;88:187-93.

22 Holt M, Rawstorne P, Wilkinson J, et al. HIV testing, gay community involvement and internet USE: social and behavioural correlates of HIV testing among Australian men who have sex with men. AIDS Behav 2012;16:13-22.

23 Hoyos J, Fernandez-Balbuena $S$, de la Fuente L, et al. Never tested for HIV in Latin-American migrants and Spaniards: prevalence and perceived barriers. J Int AIDS Soc 2013;16:18560.

24 Dedobbeleer N, Morissette P, Rojas-Viger C. Social network normative influence and sexual risk-taking among women seeking a new partner. Women Health 2005;41:63-82.

25 British HIV Association, British Association of Sexual Health and HIV, and British Infection Society. UK National Guidelines for HIV Testing 2008. British HIV Association; British Association of Sexual Health and HIV; British Infection Society, 2008.

26 Service Evaluation Economique et Santé Publique. Dépistage de linfection par le VIH en France. Stratégies et dispositif de dépistage. http://www.has-sante.fr/portail/ upload/docs/application/pdf/2009-10/argumentaire_depistage_vih_volet_2_vfv_ 2009-10-21_16-49-13 375.pdf (accessed 7 Oct 2011).

27 Center for Disease Control and Prevention. HIV testing among men who have sex with men-21 cities, United States, 2008. MMWR Morb Mortal Wkly Rep 2011:60:694-9.

28 Centers for Disease Control and Prevention. Prevalence and awareness of HIV infection among men who have sex with men- 21 cities, United States, 2008 MMWR Morb Mortal Wkly Rep 2010;59:1201-8.

29 Lucas A, Armbruster B. The cost-effectiveness of expanded HIV screening in the United States. AIDS 2013;27 